Advanced Initiation Systems Manufacturing Level 2 Milestone Completion Summary

R. Chow, M. A. Schmidt

September 1, 2010 
This document was prepared as an account of work sponsored by an agency of the United States government. Neither the United States government nor Lawrence Livermore National Security, LLC, nor any of their employees makes any warranty, expressed or implied, or assumes any legal liability or responsibility for the accuracy, completeness, or usefulness of any information, apparatus, product, or process disclosed, or represents that its use would not infringe privately owned rights. Reference herein to any specific commercial product, process, or service by trade name, trademark, manufacturer, or otherwise does not necessarily constitute or imply its endorsement, recommendation, or favoring by the United States government or Lawrence Livermore National Security, LLC. The views and opinions of authors expressed herein do not necessarily state or reflect those of the United States government or Lawrence Livermore National Security, LLC, and shall not be used for advertising or product endorsement purposes.

This work performed under the auspices of the U.S. Department of Energy by Lawrence Livermore National Laboratory under Contract DE-AC52-07NA27344. 


\section{Advanced Initiation Systems Manufacturing \\ Level 2 Milestone Completion Summary}

Project \#: FY09.0210.1

Project Name: Advanced Initiation Systems Manufacturing (AISM)

Subprogram: ADaPT Lead Site: LLNL (Participating Sites: LANL, KCP)

Milestone Description: Develop preliminary solventless process for polyimide slappers and document in a report.

\section{ABSTRACT}

The SLIP process for all-dry chip slapper coating is described. The process improvements to increase throughput and yield are reported. The AISM project has scaled up the process to uniformly coat fullsized Hermans. Rate monitoring has been installed, and control demonstrated.

\section{INTRODUCTION}

Lifetime requirements are increasing for the nuclear stockpile. Previously, a typical weapon lifetime was 20-25 years. With LEPs, lifetimes were extended by another 20-25 years. Further extensions of an extra 20 years are being considered. Extended lifetimes are driving development of processes that actively address issues of aging and compatibility. Preferred manufacturing processes use stable, compatible materials, and avoid the use of solvents, aqueous processes, and corrosive materials. Also, new processes are being developed under the AISM project to replace "sunset" slapper manufacturing technologies. One of these processes is called SLIP, an all-dry method to deposit polyimide, a generic term for Kapton ${ }^{\mathrm{TM}}$. SLIP stands for Solvent-Less vapor deposition followed by In-situ Polymerization. The SLIP process is being developed as slapper coatings on chip slappers.

Vapor-deposited Parylene-C is an alternate material under consideration for slappers. Polyimide has significant advantages over Parylene-C. Unlike Parylene-C, the SLIP process for polyimide does not require an adhesion promoter, which eliminates a wet processing step. Second, polyimide has a higher density than that of Parylene-C, a specific gravity of 1.4 vs. 1.1. Third, the mechanical properties of polyimide may be tailored to yield desirable flight characteristics. Fourth, the polyimide, Kapton, has been used in WR detonators and has survived for decades in weapon environments with no degradation of performance. Lastly, polyimide has a higher glass transition temperature than Parylene. The higher temperature survivability allows the use of other attachment options such as fluxless solders to make the joining process to the stripline dry as well.

Vapor-deposited polyimide has commercial and technological applications. There are numerous references on vapor-deposited polyimide in the open literature since it is used in the semiconductor and aerospace industries. The SLIP process was developed at LLNL as a fusion target material. Another LLNL application of the process was coating membranes for carbon sequestration tests. There is substantial experience at LLNL for the SLIP process. 


\section{EXPERIMENTAL SET-UP}

\section{SLIP Process}

The SLIP process consists of two major steps: the physical vapor deposition step and the cure step of the coating. The thickness and chemical composition of the coating is controlled in the deposition step. The final physical properties of the film are achieved during the cure step.

The deposition process occurs in a vacuum chamber. The process uses two effusion cells and a mixing nozzle. The nozzle directs both vapor fluxes towards the substrate (Herman) to be coated. Each of the effusion cells is connected to its individual temperature controller and power supply with cables that pass through a feedthrough. The effusion cells are charged with the PMDA (pyromellitic dianhydride) and ODA (oxydianiline) monomers of polyimide, respectively. The molar deposition rates are controlled by temperature; where there is an exponential (Clasius-Clayperon) relationship between temperature and the equilibrium vapor pressure of the monomer. As pressure increases, so does the deposition rate. A major part of the deposition process control procedure consists of achieving an equal number of moles of each monomer to stick to the surface. Besides the deposition rate, other variables thataffect the monomer surface concentrations are the substrate temperature, surface composition, and surface morphology.

Another purpose of the nozzle is to expose the substrate to a homogenized vapor flux of PMDA and ODA molecules. The vapors leaving the cell exit ports at the top of the mixing nozzle have a high concentration of PMDA and ODA on the left and right sides, respectively, as viewed in Figures $1 \mathrm{a}$ and $1 \mathrm{~b}$. The molecules mix during their transit towards the bottom of the nozzle. The vapor concentration gradients are smoothed by the time the flux exits the nozzle.

Figure 1a. Picture of the inside of the process chamber. The two effusion cells hang off a frame and are joined at their exit ports with a nozzle. The two effusion cells are filled with the monomers, PMDA and ODA, respectively, which make up the chemical constituents of polyimide. 


Figure 1b. Sketch of the mixing nozzle. The
monomer vapors, represented by the colored
triangles and arrows, are homogenized by the time
they reach the exit hole of the nozzle. The
substrate is exposed to a uniform vapor mixture
instead of high concentrations of PMDA and ODA
at the left and right sides of the source assembly.

A key process descriptor is the molar ratio of PMDA/ODA. An excess surface concentration of either monomer drives the formation of crystallite defects, which is the accumulation of the un-imidized monomer. The evaporated molar deposition rates are calculated after each run from the weight loss of the effusion cells incurred during a given deposition run. The coating campaign to locate the boundaries of the defectless coating regime shows that there is some latitude in the molar ratio as shown in Figure 2. The drawback with this method is that the weight changes are determined from differences of two large measurements. The material deposited in the nozzle, which also collects coating material, is not accounted for either. The calculation of the evaporated molar ratio has a large error, which is reflected in the irreproducibility of identical back-to-back runs at the same temperature set-points.

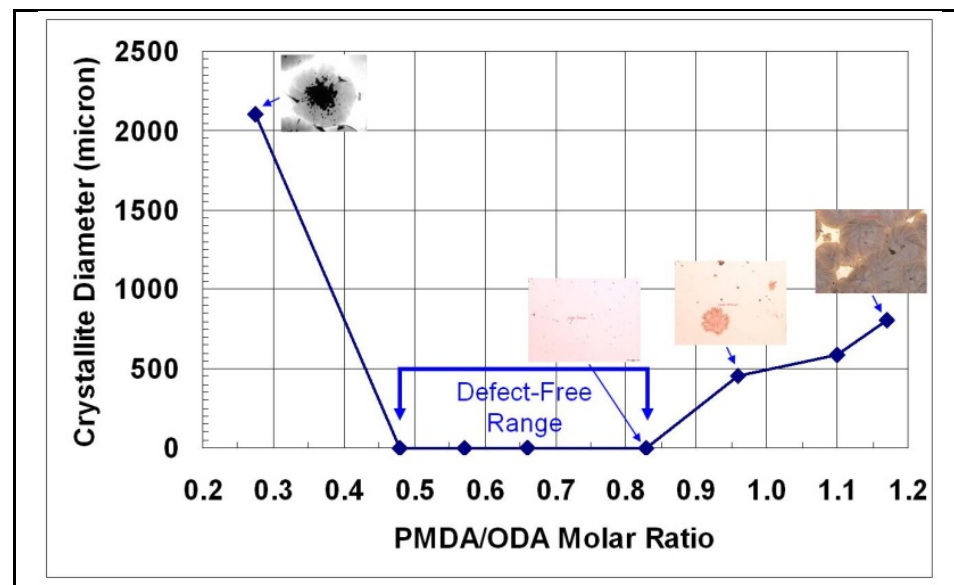

Figure 2. Crystallite defect size using high power microscopy as a function of the evaporated molar weight ratio. Crystallite-free coatings are deposited in the PMDA/ODA molar ratio range from 0.48 to 0.83 . Crystallite defects formed from excessive monomer concentration. As the monomers polymerize and imidize, the unreacted monomers crystallize into coating defects. The defects are larger with greater non-stoichiometry ratio.

Temperature curing completes the chemical reactions that give the coating its final mechanical and electrical properties. $\mathrm{KBr}$ samples ( $13 \mathrm{~mm}$ diameter $\mathrm{x} 1 \mathrm{~mm}$ thick) were coated with $0.4 \mu \mathrm{m}$ of polyimide. One sample was kept uncured and the others were cured at $100 \mathrm{C}, 150 \mathrm{C}, 200 \mathrm{C}, 300 \mathrm{C}$ and $390 \mathrm{C}$, respectively. The ramp-up and -down times were $1 \mathrm{hr}$ each, and the soak time was $2 \mathrm{hrs}$. FTIR scans were performed with a Perkin-Elmer Spectrum 2000 spectrophotometer to observe changes of the $13801 / \mathrm{cm}$ peak, indicative of the imidization reaction. The results are plotted in Figure 3 , along 
with some mechanical properties of the coating. The changes in hardness and Elastic Modulus correlate inversely with the degree of imidization (DOI) at temperatures between $100 \mathrm{C}$ and $200 \mathrm{C}$. Curing at temperatures above $200 \mathrm{C}$ does not significantly change the DOI or the aforementioned mechanical properties. However the surface roughness increases at temperatures $>200 \mathrm{C}$. The surface roughening is attributed to thermal degradation of the coating. The optimal cure temperature is $\approx 200 \mathrm{C}$, which is similar to that of the conventional wet-cast Kapton. The cure temperature and mechanical properties indicate that the SLIP process produces polyimide similar to that of the conventional method. For reference, the mechanical properties of Parylene- $C$ are indicated by the open symbols at room temperature.

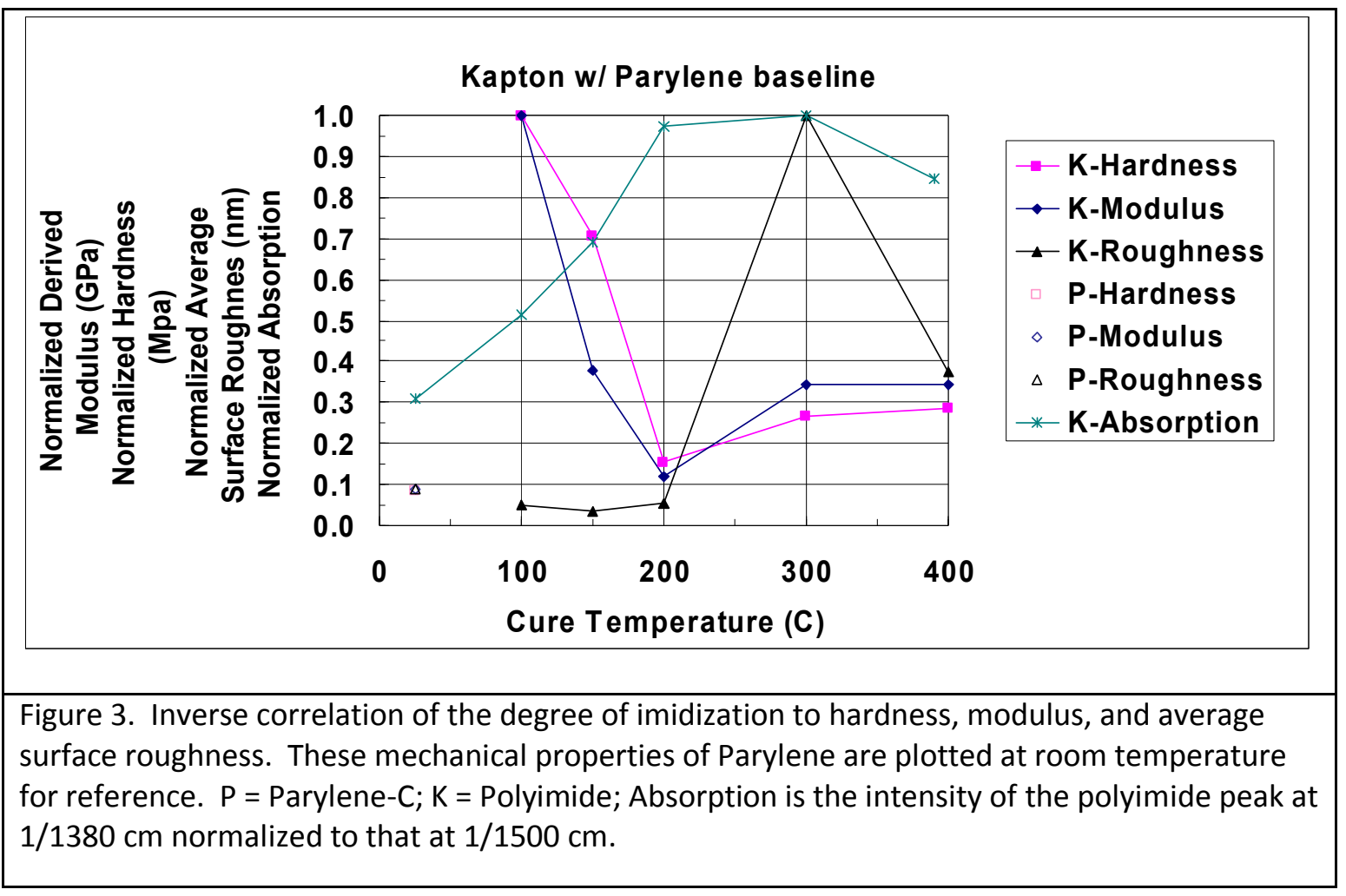

Results and Discussion of SLIP Process Improvements

There were two major improvements to the SLIP process. One was to increase throughput with better thickness uniformity and thereby coating more parts per run. The other was to monitor the vapor flux to control the chemical concentrations of PMDA/ODA, and not depend on temperature as a means to control deposition chemistry.

There is a rapid thickness drop at the edges of the Herman because the current SLIP system (machine, effusion cells, and nozzles) was designed for fusion targets, which are on the order of $1 \mathrm{~mm}$ in diameter. The current system, therefore, has severe thickness non-uniformity for larger parts like a Herman that the chip slappers are built on. As illustrated by Figure 4, the Herman is over two orders of magnitude larger than a fusion target. The initial attempt to improve thickness uniformity was to increase the 
separation distance between the mixing nozzle and the substrate. Using this approach, the system was able to coat a quartered Herman with acceptable thickness uniformity. There was limited success due to the volume restrictions of the small process chamber that prevented any further separation.

\begin{tabular}{|l|l|}
\hline & $\begin{array}{l}\text { Figure 4. The red dot in the center of the chip } \\
\text { slapper Herman represents the size of a fusion } \\
\text { target relative to that of the Herman. The dashed } \\
\text { blue line outlines the area where the chip slapper } \\
\text { device is patterned. The coating thickness falls off } \\
\text { rapidly at the edges of the Herman since the SLIP } \\
\text { system was designed to only coat target-sized } \\
\text { substrates. }\end{array}$ \\
\hline
\end{tabular}

A SLIP process model was created in order to design nozzles that would not only mix the vapor but also improve the thickness uniformity over the entire device area of a Herman. There are two pressure regimes of interest in the SLIP coating process. There is a relatively high pressure regime in the nozzle and a low pressure regime between the nozzle exit and the substrate. A Direct Simulation Monte Carlo (DSMC) method was used to simulate the gas properties in the nozzle. The pressure in the nozzle is not high enough for a continuum solution (e.g. Navier-Stokes equations) to be physically accurate. The DSMC method accurately captures non-continuum, non-equilibrium gas dynamics by solving the Boltzmann equation in a probabilistic manner using discrete simulation particles to represent the gas behavior. The gas transport in the low pressure regime is approximated by the collisionless Boltzmann equation. The collisionless Boltzmann equation is solved using a test particle Monte Carlo (MC) method. The test particle MC method uses much less computational time compared to the DSMC method. A DSMC solution is generated for the nozzle to determine the gas properties and distribution of molecular trajectory angles at the exit. The DSMC solution is then used as the initial conditions of the test particle MC method to propagate the monomer molecules from the nozzle to the substrate. The incident molecular flux is then recorded across the substrate to determine the expected thickness uniformity, composition, and rate of the deposited film.

The SLIP coating process was simulated first with and without a nozzle to validate the model. The model predicts and visual inspection of the coatings shows that the vapor flux iswell-mixed at the nozzle exit. The model predicted and thickness measurements showed that without the nozzle, there is a concentration gradient corresponding to the offset of PMDA to the left and ODA to the right of the substrate.

A number of simulations were performed on high-uniformity nozzles (HUNs) shaped as shown in Figure 5. The HUN showerhead concept is to direct the vapor away from the center of the substrate, where the coating is thickest, and towards the edges, where the coating is thinnest. The simulations 
modeled the effects of the vapor duct angle, length-to-radius aspect ratio of the ducts, and the vapor pressure inside the HUN. The primary driver for thickness uniformity improvements is the duct angle. Nozzles were made at various angles and used to deposit coatings for thickness uniformities measurements. The normalized thickness uniformities are plotted in Figure 6 as a function of substrate diameter and duct angle. There was no noticeable improvement as the angle increased from zero to $15^{\circ}$. Noticeable improvements came at the $30^{\circ}$ and $45^{\circ}$ duct angles. The current system with the $45^{\circ}$ HUN now has the capability of coating an entire Herman's worth of chip slappers with a thickness uniformity of $\pm 11 \%$.

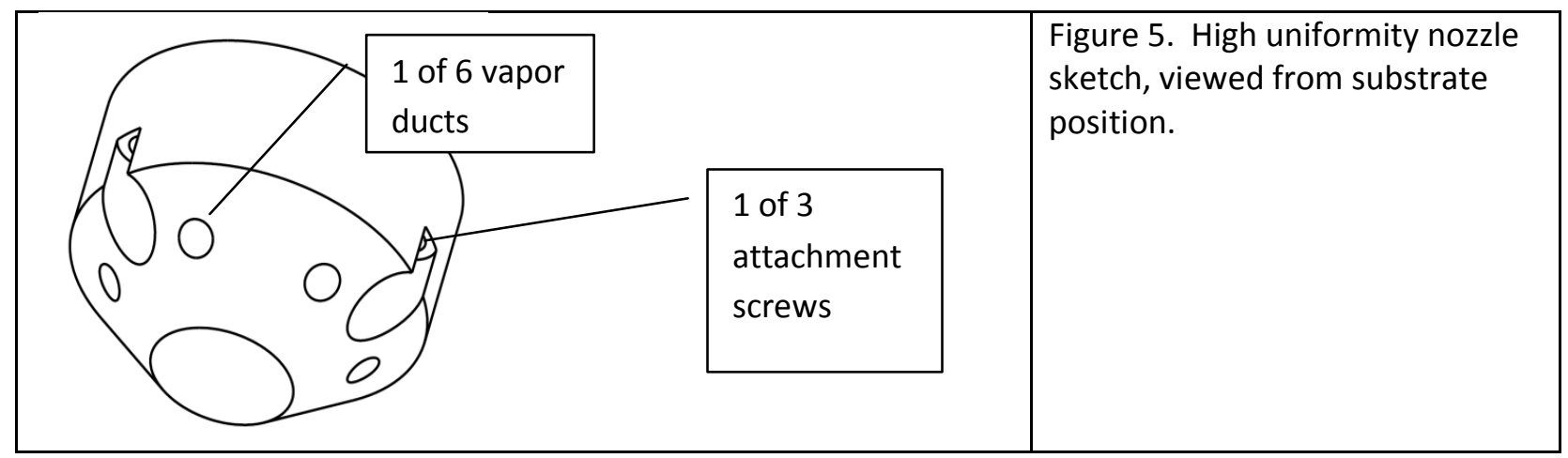

The improved uniformity comes at the cost of lower deposition rates. The $45^{\circ} \mathrm{HUN}$ has an average deposition rate that is $4 \mathrm{x}$ slower than that of the nozzle with a zero degree duct angle. Other conclusions from the modeling effort are that the higher pressures affect thickness uniformity at angles $<15^{\circ}$ and aspect ratios affect thickness uniformity at angles $<20^{\circ}$ and at lower pressures.

\begin{tabular}{|c|c|}
\hline \begin{tabular}{l|l|l|l|}
\multicolumn{1}{c}{ Comparison per Normalized Averages } \\
\end{tabular} & $\begin{array}{l}\text { Figure } 6 . \text { Normalized thickness } \\
\text { uniformity as function of substrate } \\
\text { diameter and duct angle on the HUN. }\end{array}$ \\
\hline
\end{tabular}

The SLIP process for depositing thin coatings did not appear to be reproducible. Even in back- to- back runs at the same temperature set-point, there were variations in the molar weight ratios, average thicknesses, and coating stress. To address these process variations, rate control diagnostics were added onto both cells. The diagnostic hardware included power supplies for the effusion cells (Figure 7a), Inficon rate controller (Figure 7b), and quartz crystal sensors (Figure 7c). The sensors and vapor sampling ports were positioned on opposing sides to minimize flux cross-talk. Preliminary tests were 
performed to determine an appropriate port diameter to sample the vapor flux. The smaller diameters prolonged the sensor life and had less impact on the vapor flowing out through the main cell orifice. However, the smaller diameters were extremely difficult to check for clogging. A 0.0135" diameter was selected, which is $12 \%$ of the area of the cell orifice. An 80 -gauge drill bit is used as a reamer tool for the sampling ports.

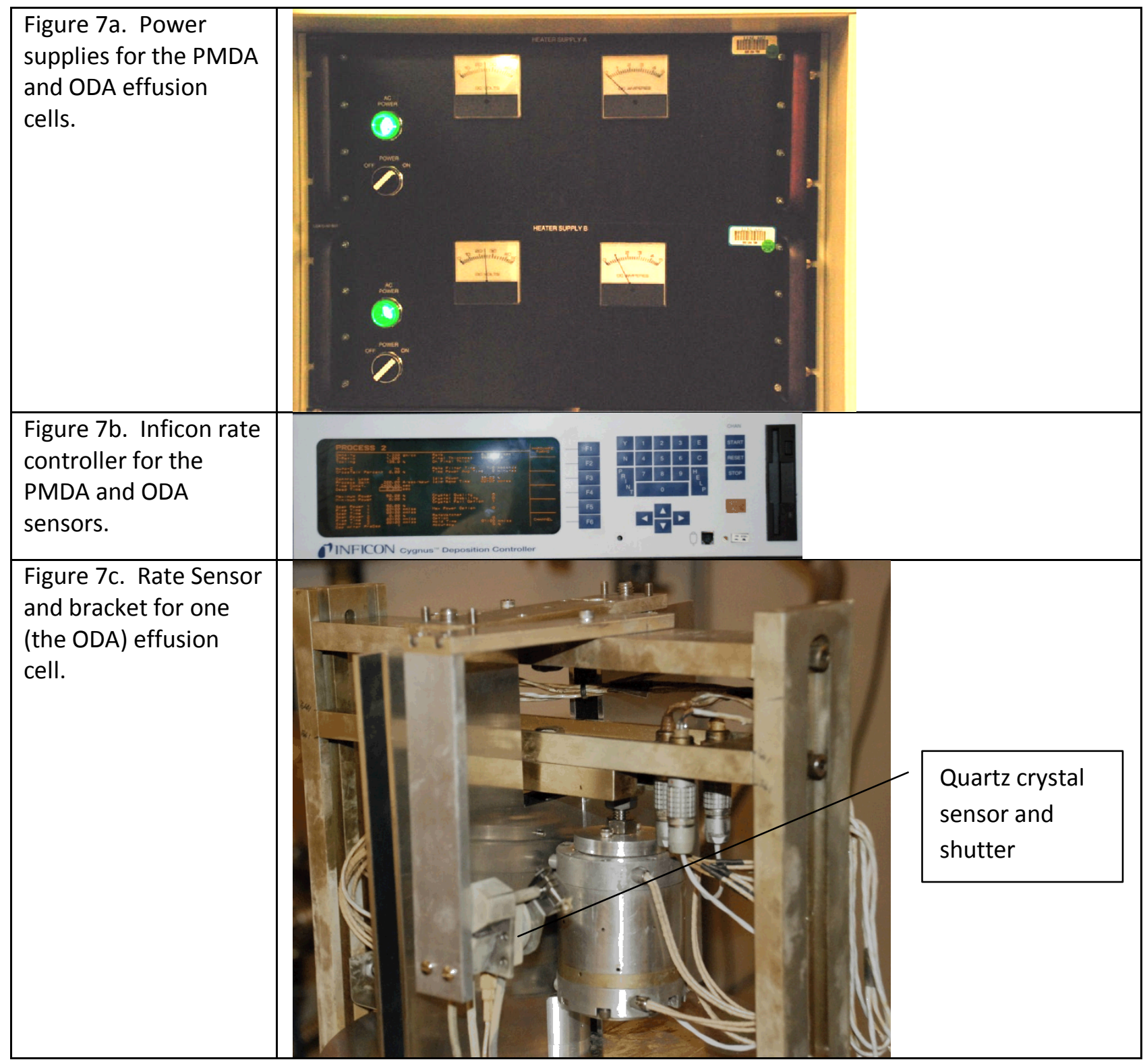

In Figure 8, the time profiles of cell temperature, rate, and power are plotted from a representative run. Both cells were powered on under temperature control where at elapsedtime $=0$, the temperatures were at room temperature and the power demand is at zero. At a time shortly after $t=0$, the power supplied went to $100 \%$. The temperature and power time profiles are plotted versus the right axis. The rate profile is plotted on the left axis. The cell temperatures increased with time and stabilized at $t \approx 40$ 
min. However, the respective rates can take much longer to stabilize afterwards - up to 30 minutes for PMDA and 90 minutes for ODA. The rate ratio of PMDA/ODA is plotted in red and values are on the left axis. The rate ratio shows that the vapor stoichiometry exiting the mixing nozzle can vary up to $5 x$ in the course of a one hour run. The elapsed time to reach rate stability may be another likely cause of the process irreproducibility since it depends on when the substrate shutter opens to start the coating deposition. As reference, the typical operating procedure, before having the rate diagnostic capabilities, was to wait at least an hour after power was turned on before starting the coating run.

Another observation is that although the temperature controls of the cells are within $\pm 0.5^{\circ} \mathrm{C}$, the rates have a drift component. It is expected that as the charge depletes, the rate decreases. This is observed in this run with the ODA cell. However, the PMDA rate increases with run-time by about $8 \%$ over a $2 \mathrm{hr}$ period. The rate spikes at 188 minutes are where the shutters were intentionally closed to the quartz crystals as a functionality test. The other smaller rate spikes, noticeably at 220 minutes, could be due to electrical or process (charge spitting) noise. The major conclusion from data as shown in Figure 8 is that rate monitoring will be essential for process control.

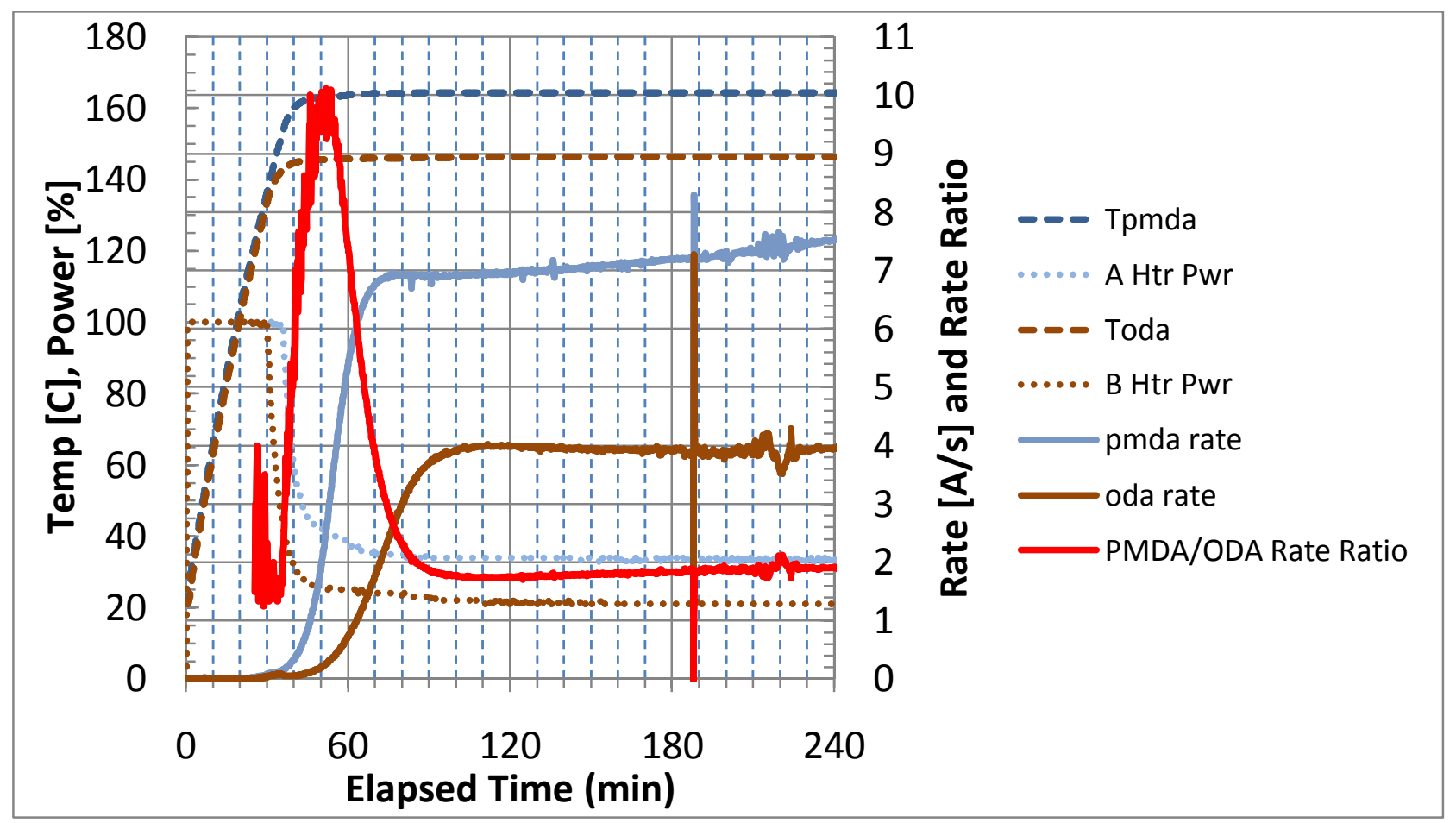

Figure 8. Rate monitoring of the cells under temperature control; Run \#R100414a. The color scheme: ODA is brown and PMDA is blue. The line style scheme: Temperature is dashed, Power supplied to cells is dotted, Rate and the Rate Ratio is solid.

Two options were considered for improved process control. The first option is to operate under temperature control and to have an operator adjust the temperature on an hourly period to maintain a constant rate. This is not the preferred method for operation. The second option is to use a rate controller with control parameter ranges suitable for thermal systems that are relatively slow 
responding and are heated to relatively low process temperatures. The next phase for process improvement was to move from rate monitoring to rate control of the effusion cells.

After many trials, the best rate control still had unacceptable oscillatory responses of $25 \%$ about the rate set-point (Figure 9a). The lack of rate control was attributed to the fact that the effusion cells operate at temperatures below $500^{\circ} \mathrm{C}$ and therefore are very thermally slow-responding systems. The heat has to be conducted away not only from the metal parts of the cell but also the monomer charge. A rate controller with appropriate range of PID control parameters was found and installed. Another round of trials to establish the control parameters were undertaken. As shown in Figure 9b, rate control, with one cell operating, within $1.7 \%$ was achieved.

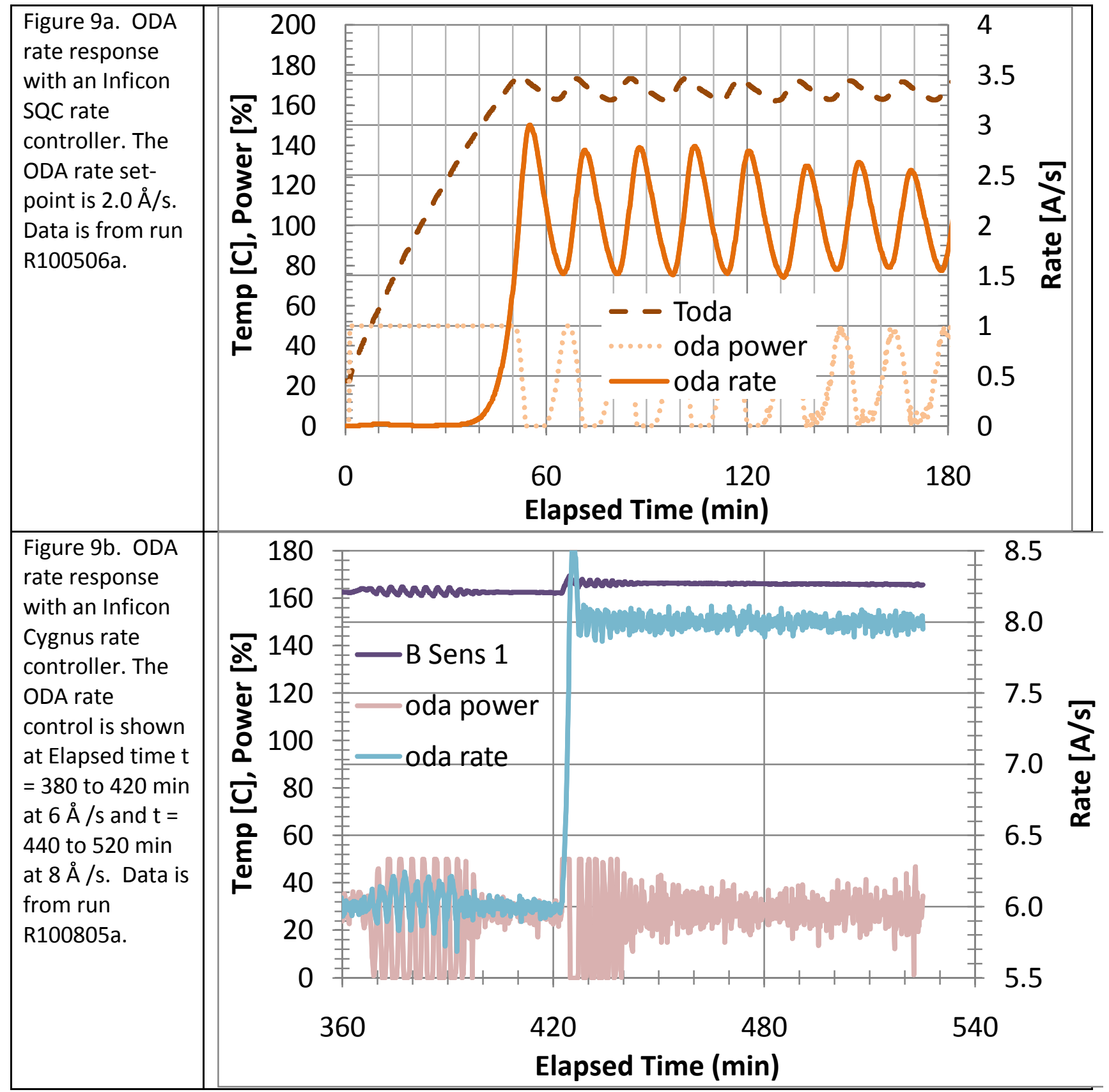


During the rate control tests, there appeared to be crosstalk between the vapor from one cell reaching the sensor of the opposing cell. The vapor leak was traced to the area where the HUN attaches to the two effusion cell exit ports. The solution was to modify the HUN with an internal O-ring groove. The Oring would block the vapor from seeping between the HUN and the cell exit ports to the sensors. The remaining vapor leak came between the surfaces where the two cell exit ports touch each other. The solution here was to add two parallel vertical shields that would direct the vapor away from the sensors and to the edge of the chamber where it would be pumped. Figures 10a and 10b are pictures of the modified HUN and vertical shields.

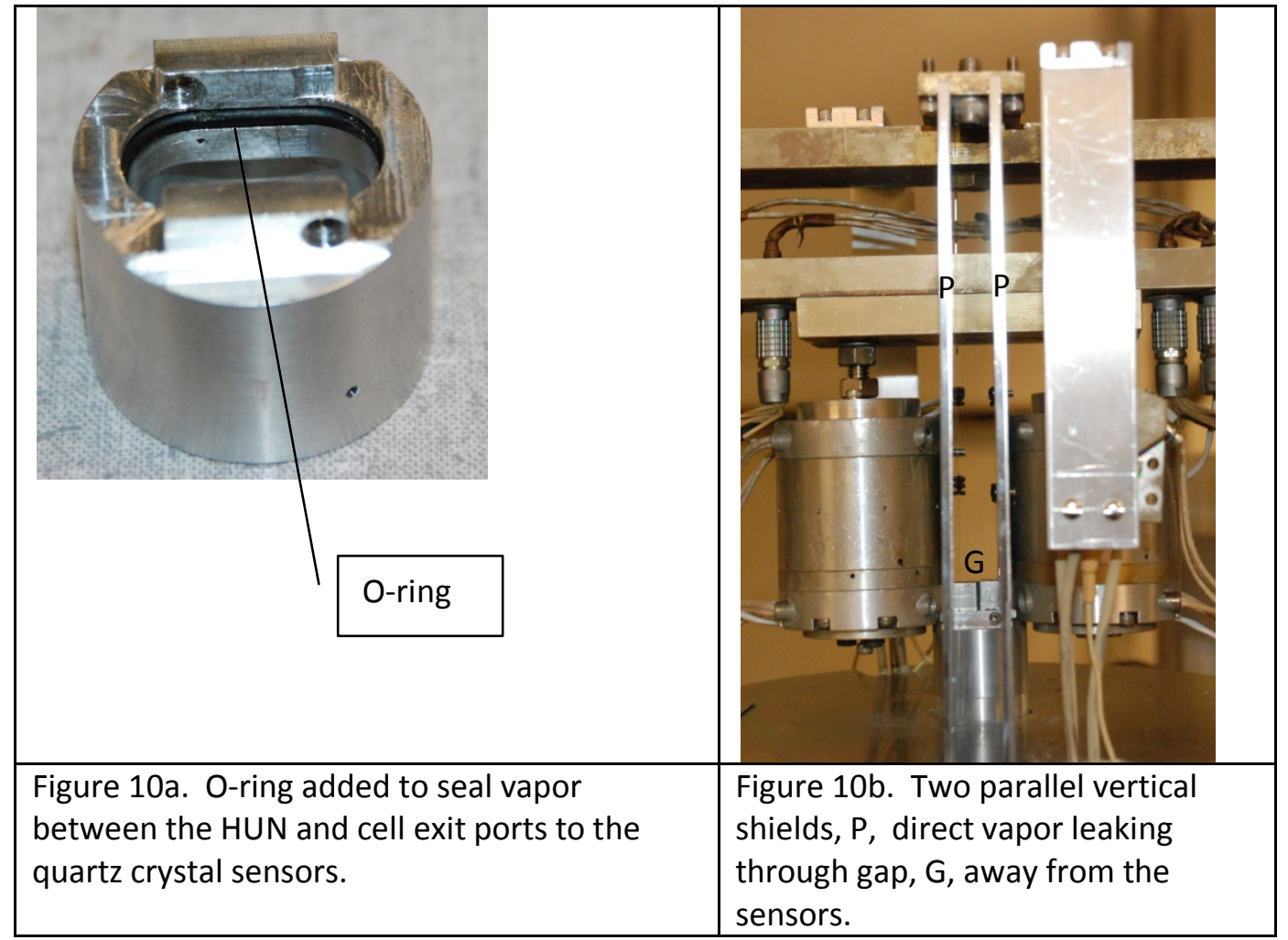

\section{SUMMARY}

The SLIP polyimide has desirable properties for low-energy chip slappers

1. The SLIP process is solvent-free which makes the coatings more compatible with HE and less subject to degradation with age. In contrast, other potential slapper coatings, such as Parylene or wet-cast Kapton, use solvents in their manufacturing processes.

2. The SLIP coating withstands higher temperatures than Parylene-C, which helps in the use of fluxless solders for attaching chips to cables.

The SLIP Process Improvements 
1. The elastic modulus, hardness, and surface roughness as a function of cure temperature were measured for SLIP coatings near the sublimated molar ratio of 1.

2. Simulated the SLIP process and obtained thickness uniformity to coat full-sized Hermans.

3. Installed rate monitoring diagnostics to control the coating process reproducibility.

4. Determined that deposition rate equilibrium can lag the temperature equilibrium by as much as 30 minutes.

5. Determined that deposition rate equilibrium is not achieved by temperature equilibrium.

6. Eliminated vapor cross-talk between the rate sensors.

7. Demonstrated rate control with the ODA cell.

Report Originators: Robert Chow and Mark Schmidt

Report Date: September 30, 2010

Auspices: Lawrence Livermore National Laboratory is operated by Lawrence Livermore National Security, LLC, for the U.S. Department of Energy, National Nuclear Security Administration under Contract DE-AC52-07NA27344. 